\section{BRAZIULIAN JOURNAL}

OF MEDICAL AND BIOLOGICAL RLSH.ARCH

www.bjournal.com.br
ISSN 0100-879X

Volume 43 (02) 124-225 February 2010

BIOMEDICAL SCIENCES

AND

CLINICAL INVESTIGATION

Braz J Med Biol Res, February 2010, Volume 43(2) 139-149

Anti-parasitic action and elimination of intracellular Toxoplasma gondii in the presence of novel thiosemicarbazone and its 4-thiazolidinone derivatives

C.S. Carvalho, E.J.T. de Melo, R.P. Tenório and A.J.S. Góes

The Brazilian Journal of Medical and Biological Research is partially financed by
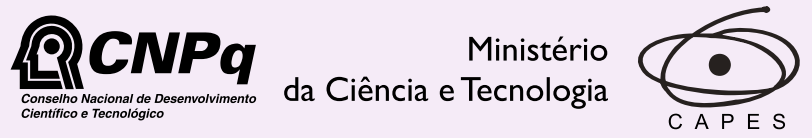

Ministério da Educação

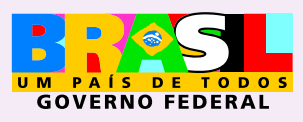

Institutional Sponsors 


\title{
Anti-parasitic action and elimination of intracellular Toxoplasma gondii in the presence of novel thiosemicarbazone and its 4-thiazolidinone derivatives
}

\author{
C.S. Carvalho1 ${ }^{1}$, E.J.T. de Melo ${ }^{1}$, R.P. Tenório ${ }^{2}$ and A.J.S. Góes ${ }^{2}$ \\ ${ }^{1}$ Setor de Toxicologia Celular, Laboratório de Biologia Celular e Tecidual, \\ Universidade Estadual do Norte Fluminense, Rio de Janeiro, RJ, Brasil \\ ${ }^{2}$ Departamento de Antibióticos, Universidade Federal de Pernambuco, Recife, PE, Brasil
}

\begin{abstract}
Toxoplasma, which infects all eukaryotic cells, is considered to be a good system for the study of drug action and of the behavior of infected host cells. In the present study, we asked if thiosemicarbazone derivatives can be effective against tachyzoites and which morphological and ultrastructural features of host cells and parasites are associated with the destruction of Toxoplasma. The compounds were tested in infected Vero cell culture using concentration screens ( 0.1 to $20 \mathrm{mM}$ ). The final concentration of $1 \mathrm{mM}$ was chosen for biological assay. The following results were obtained: 1) These new derivatives decreased $T$. gondii infection with an in vitro parasite IC50\% of 0.2-0.7 mM, without a significant effect on host cells and the more efficient compounds were 2, 3 (thiosemicarbazone derivatives) and 4 (thiazolidinone derivative); 2) The main feature observed during parasite elimination was continuous morphological disorganization of the tachyzoite secretory system, progressive organelle vesiculation, and then complete disruption; 3) Ultrastructural assays also revealed that progressive vesiculation in the cytoplasm of treated parasites did not occur in the host cell; 4) Vesiculation inside the parasite resulted in death, but this feature occurred asynchronously in different intracellular tachyzoites; 5) The death and elimination of $T$. gondii was associated with features such as apoptosis-like stage, acidification and digestion of parasites into parasitophorous vacuoles. Our results suggest that these new chemical compounds are promising for the elimination of intracellular parasites by mainly affecting tachyzoite development at $1 \mathrm{mM}$ concentration for $24 \mathrm{~h}$ of treatment.
\end{abstract}

Key words: Acid [2-(benzylidene-hydrazone)-4-oxo-1,3-thiazolidin-5-yl]acetic; $\rho$-chloro-benzaldehyde thiosemicarbazone; Tachyzoite; Toxoplasma

\section{Introduction}

Toxoplasma gondii, the etiological agent of toxoplasmosis, is a parasite that establishes a safe niche in the cytoplasm of all host cells - the parasitophorous vacuole (PV). This environment surrounds the parasite and has a close association with the host cell mitochondrial membrane and endoplasmic reticulum $(1,2)$, but is free of host cell protein (2). Thus, the PV is not acidified and is resistant to fusion with host endocytic and lysosomal compartments $(3,4)$. Once inside the cell, T. gondii begins to grow and divide by endodyogeny $(5,6)$ and while developing it captures nutrients such as phospholipids (7), cholesterol, glucose, and purine nucleosides from the host cell $(8,9)$.

The T. gondii-host cell interaction is a good system to study the action of drugs and the behavior of infected host cells and cell cycle inhibition has been shown to be a useful target for drug development (10). We initially used an anti-proliferative drug hydroxyurea $(\mathrm{HU})$ against intracellular parasites such as T. gondii, Leishmania amazonensis and Trypanosoma cruzi. This drug arrested the replication of these microorganisms, resulting in parasite elimination and reduced infection in vitro $(11,12)$.

$\mathrm{HU}$ is a urea derivative, which has the basic structure $\mathrm{HONH}-\mathrm{CO}-\mathrm{NH}_{2}$, and thiosemicarbazone has the structure $\mathrm{R}_{2} \mathrm{C}=\mathrm{N}-\mathrm{NH}-\mathrm{CS}-\mathrm{NHR}$. $\mathrm{HU}$ and thiosemicarbazones are known to inhibit the cell cycle in the G1/S phase, mainly inactivating ribonucleotide reductase, an enzyme that mediates the conversion of ribonucleotides to deoxyribonucleotides (13). Recently, we demonstrated that TSC and

Correspondence: E.J.T. de Melo, Setor de Toxicologia Celular, Laboratório de Biologia Celular e Tecidual, Universidade Estadual do Norte Fluminense, 28013-602 Rio de Janeiro, RJ, Brasil. Fax: +55-22-2739-7178. E-mail: ejtm1202@gmail.com

Received May 19, 2009. Accepted October 1, 2009. Available online November 6, 2009. Published February 1, 2010. 
analog compounds, 4-thiazolidinones (4-TZD), decreased in vitro infection and eliminated intracellular T. gondii (14). This study used TSC substituted at the arylhydrazone moiety with a nitro substituent at the ortho, meta and para positions, and 4-TZD substituted at the $N-3$ position with a phenyl, methyl and hydrogen substituent, and with the same groups at the arylhydrazone moiety. Compounds bearing a heterocyclic 4-TZD have high biological activity including antimicrobial (15), antiviral (16), anti-inflammatory (17), hypnotic (18), and antiprotozoal activity $(19,20)$. Both TSC and TZD compounds reduced infection, but the 4-TZD compounds were more efficient (14).

The radical change in the molecule's structure should improve efficacy of the molecules, increasing their antiparasitic or anti-proliferative effect. Thus, the aim of the present study was to evaluate the effects of new TSC and TZD compounds that have one arylhydrazone moiety with a hydrogen, chlorine and nitro group (Figure 1) against intracellular T. gondii. It is important to point out that the standard therapies for toxoplasmosis - the combination of pyrimethamine and sulfadiazine, clindamycin, azithromycin, or atavaquone $(21,22)$ - do not eliminate the intracellular parasite and are often associated with severe side effects (23). This has increased the need to develop new drugs. Furthermore, we report the morphological features of parasite destruction in the presence of the drugs in order to identify the cellular mechanism associated with the microbicidal effects of the compounds tested.

\section{Material and Methods}

\section{Host cells}

Vero cells (kidney fibroblasts of the African monkey) were grown in plastic Falcon flasks $\left(25 \mathrm{~cm}^{2}\right)$ containing medium 199 (Sigma, USA) with 5\% fetal calf serum (FCS; Gibco, USA), and were trypsinized when the cell density approached a semi-confluent monolayer. One day before use in the experiments, approximately $3 \times 10^{4}$ cells were placed on Linbro 24-well tissue plates containing a sterile round coverslip, or were placed in $25-\mathrm{cm}^{2}$ flasks (3-5 x 105/ flasks), and maintained at $37^{\circ} \mathrm{C}$ overnight in a $5 \% \mathrm{CO}_{2}, 95 \%$ air atmosphere.

\section{Parasites}

Tachyzoites from the virulent RH strain of T. gondii were maintained by intraperitoneal passages in Swiss mice and were collected in phosphate-buffered saline (PBS), $\mathrm{pH} 7.2$, $48 \mathrm{~h}$ after infection. The ascitic fluid obtained from infected mice, which contained the parasites, was collected and centrifuged at $300 \mathrm{~g}$ for $10 \mathrm{~min}$. The pellet obtained was washed with PBS, pH 7.2, and suspended to a density of $10^{7}$ parasites $/ \mathrm{mL}$ in medium 199 without FCS. The parasites were used about 30 min after removal from infected animals, and viability was evaluated using a dye-exclusion test with $0.2 \%$ Trypan blue (w/v). All procedures for the use of animals followed the Ethical Guidelines for Animal Experimentation of the Universidade Estadual do Norte Fluminense.

\section{Host cell-parasite interaction}

Parasites suspended in medium 199 were incubated for $2 \mathrm{~h}$ in the presence of Vero cells using a 5:1 parasite-host cell ratio. After incubation, the cells were washed twice with medium 199 to remove extracellular parasites, and incubated for $24 \mathrm{~h}$ at $37^{\circ} \mathrm{C}$. The compounds described below were then added to the cultures.

\section{Chemistry}

The synthesis of TSC (1-3) and 4-TZD (4-6) derivatives (Figure 1) followed the method described by Tenório et al. (14). The chemical names of the compounds are the following: compound 1 (benzaldehyde thiosemicarbazone); compound 2 (para-chloro-benzaldehyde thiosemicarbazone); compound 3 (para-nitro-benzaldehyde thiosemicarbazone); compound 4 (acid [2-(benzylidene-hydrazone)-4-oxo1,3-thiazolidin-5-yl]acetic); compound 5 (acid [2-chloro(benzylidene-hydrazone)-4-oxo-1,3-thiazolidin-5-yl]acetic); compound 6 (acid [2-para-(nitro-benzylidene-hydrazone)-4oxo-1,3-thiazolidin-5yl]acetic). Briefly, the TSC 1, 2, and 3 were obtained by reaction between the thiosemicarbazide and the respective benzaldehydes substituted in acid medium under reflux, at satisfactory yields (56-96\%). These compounds were then cyclized with excess maleic anhydride in dry toluene and $\mathrm{N}, \mathrm{N}$-dimethylformamide to yield the respective 4-TZD derivatives 4, 5, and 6 (52-96\%). All compounds are stable both in the solid and solution state. Spectroscopic data for these compounds were consistent with their structure. HU was obtained from Sigma and sulfadiazine from Merck (USA).

\section{Incubation with the compounds}

After incubation of the cells with the parasites for $24 \mathrm{~h}$, the cells were incubated with the compounds described above. TSC and TZD and derivatives were used at a final concentration of $1 \mathrm{mM}$ for biological assays. This concentration was selected according to the concentration screen ( 0.1 to $20 \mathrm{mM}$ ), and following preliminary experiments and previous studies carried out by Tenório et al. (14). HU and sulfadiazine were used as the reference drugs, at 4 and 1

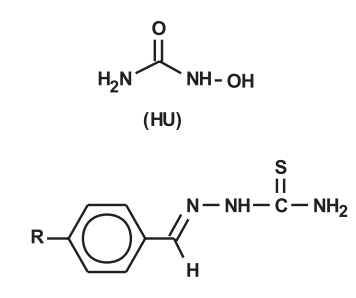

(1) $\mathrm{R}=\mathrm{H},(2) \mathrm{R}=\mathrm{Cl},(3) \mathrm{R}=\mathrm{NO}_{2}$

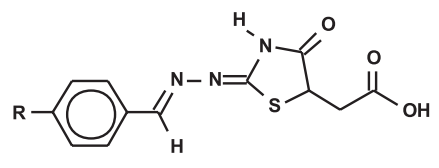

(4) $\mathrm{R}=\mathrm{H},(5) \mathrm{R}=\mathrm{Cl},(6) \mathrm{R}=\mathrm{NO}_{2}$
Figure 1. Structures of anti-Toxoplasma drugs hydroxyurea (HU), thiosemicarbazones (1-3) and 4-thiazolidinones (4-6). 
$\mathrm{mM}$, respectively, according our previous data $(11,14)$. All drugs were dissolved in DMSO and added to medium 199. DMSO was used at a final concentration of $1 \%(\mathrm{v} / \mathrm{v})$, which was not toxic for Vero cells or intracellular T. gondii. Infected cells were incubated in the presence of $\mathrm{HU}$ and compounds 1-6 for 12 or $24 \mathrm{~h}$. In order to determine the reversible effect of the compounds on the parasites, the medium containing the drug was removed and the cultures were incubated for $24 \mathrm{~h}$ with fresh medium 199 supplemented with 5\% FCS, at $37^{\circ} \mathrm{C}$ in $5 \% \mathrm{CO}_{2}$. Trypan blue $(0.2 \% \mathrm{w} / \mathrm{v})$ was used to assay the viability of treated and untreated host cells.

\section{Morphological analyses and quantification}

For morphological analyses, infected and treated cultures were rinsed with $\mathrm{PBS}, \mathrm{pH} 7.2$, at $37^{\circ} \mathrm{C}$. Cells were then fixed with Bouin's solution for 15 min and stained with Giemsa $(10 \% \mathrm{v} / \mathrm{v})$, for $2 \mathrm{~h}$ at room temperature. All preparations were examined using a Zeiss Axioplan photomicroscope equipped with a $63 \mathrm{X}$ objective. The images were obtained with the Analysis System software (Germany).

At least 300 cells in the same monolayer were scored at 400X magnification for each drug and each time tested. The following parameters were analyzed in treated and untreated cultures: a) normal intracellular parasites, which have a crescent shape; b) infected cells containing parasites with normal morphology; c) infected cells containing parasites with altered morphology; d) parasitophorous vacuoles containing altered parasites. Finally, the LD50 values of all compounds for host cells and intracellular tachyzoites were obtained after $24 \mathrm{~h}$ of exposure at the concentration of 0.1-20 mM.

Statistical analysis was performed by twoway ANOVA (Graph Pad Prism). P values of $<0.05$ were considered to be significant. The data reported here are representative of three experiments performed in quadruplicate.

\section{Ultrastructural analyses}

For ultrastructural analyses, Vero cells were placed in culture flasks $\left(75 \mathrm{~cm}^{2}\right)$ and infected with parasites as described before. After incubation with the drugs, the cultures were washed with PBS at $37^{\circ} \mathrm{C}$ and fixed at room temperature in a solution containing $1 \%$ glutaraldehyde, $4 \%$ paraformaldehyde, $5 \mathrm{mM}$ $\mathrm{CaCl}_{2}$, and $5 \%$ sucrose in $0.1 \mathrm{M}$-cacodylate buffer, pH 7.2. Cells were then post-fixed for $1 \mathrm{~h}$ in a solution containing $2 \% \mathrm{OsO}_{4}, 0.8 \%$ potassium ferrocyanide, and $5 \mathrm{mM} \mathrm{CaCl}_{2}$ in 0.1 M-cacodylate buffer, $\mathrm{pH}$ 7.2. Samples were rinsed with $0.1 \mathrm{M}$ cacodylate buffer, $\mathrm{pH}$ 7.2 , dehydrated in acetone and embedded in PolyBed (Polyscience Inc., USA). Thin sec- tions were stained with uranyl acetate and lead citrate and observed with a Zeiss 900 Transmission Electron Microscope at $80 \mathrm{kV}$ acceleration.

\section{Vacuolar acidification}

To examine vacuolar acidification, treated and untreated infected cultures were incubated with a solution of acridine orange (AO) dye (Sigma), at a final concentration of $5 \mu \mathrm{g} / \mathrm{mL}$ for $20 \mathrm{~min}$ (24). Live samples were then washed with culture medium and examined under a Zeiss Confocal Laser Scan Microscope using a 488-nm or 543-nm argon laser.

\section{Results}

Vero cells infected with $T$. gondii for 24 h presented an established infection with a high number of intravacuolar tachyzoites. These infected cultures were then incubated in the presence of TSC and 4-TDZ for 12 and $24 \mathrm{~h}$. Quantitative and morphological analyses were performed to observe the anti- $T$. gondii action of the drugs.

According to Tables 1 and 2, the action of all compounds

Table 1. Cytotoxic effects of compounds 1-6, hydroxyurea and sulfadiazine on Vero cells and intracellular Toxoplasma gondii tachyzoites.

\begin{tabular}{|c|c|c|c|c|c|c|}
\hline \multicolumn{7}{|c|}{ Mean number of host cells $\left(x 10^{5}\right)$} \\
\hline \multirow[t]{2}{*}{ Compound } & \multirow[t]{2}{*}{ Untreated } & \multicolumn{5}{|c|}{ Treated } \\
\hline & & $0.1 \mathrm{mM}$ & $1 \mathrm{mM}$ & $4 \mathrm{mM}$ & $8 \mathrm{mM}$ & $20 \mathrm{mM}$ \\
\hline 1 & $430 \pm 6$ & $379 \pm 12$ & $318 \pm 4$ & $511 \pm 4$ & $390 \pm 1$ & $78 \pm 9$ \\
\hline 2 & $120 \pm 9$ & $140 \pm 8$ & $130 \pm 10$ & $194 \pm 7$ & $143 \pm 3$ & $80 \pm 7$ \\
\hline 3 & $158 \pm 9$ & $165 \pm 9$ & $144 \pm 5$ & $206 \pm 4$ & $169 \pm 7$ & $87 \pm 6$ \\
\hline 4 & $146 \pm 2$ & $160 \pm 9$ & $147 \pm 8$ & $224 \pm 5$ & $290 \pm 6$ & $65 \pm 9$ \\
\hline 5 & $163 \pm 8$ & $180 \pm 9$ & $144 \pm 16$ & $270 \pm 7$ & $158 \pm 2$ & $172 \pm 4$ \\
\hline 6 & $199 \pm 5$ & $168 \pm 7$ & $155 \pm 7$ & $210 \pm 6$ & $180 \pm 1$ & $182 \pm 6$ \\
\hline Hydroxyurea & $205 \pm 9$ & $168 \pm 5$ & $180 \pm 9$ & $113 \pm 6$ & $167 \pm 1$ & $180 \pm 7$ \\
\hline Sulfadiazine & $98 \pm 7$ & $130 \pm 2$ & $136 \pm 2$ & $79 \pm 2$ & $54 \pm 1$ & $14 \pm 2$ \\
\hline \multicolumn{7}{|c|}{ Mean number of intracellular parasites $\left(\times 10^{5}\right)$} \\
\hline \multirow[t]{2}{*}{ Compound } & Untreated & \multicolumn{5}{|c|}{ Treated } \\
\hline & & $0.1 \mathrm{mM}$ & $1 \mathrm{mM}$ & $4 \mathrm{mM}$ & $8 \mathrm{mM}$ & $20 \mathrm{mM}$ \\
\hline 1 & $2381 \pm 53$ & $2450 \pm 16$ & $29 \pm 2$ & $19 \pm 7$ & $7 \pm 3$ & 0 \\
\hline 2 & $584 \pm 20$ & $602 \pm 7$ & $13 \pm 2$ & $2 \pm 1$ & 0 & 0 \\
\hline 3 & $1058 \pm 57$ & $989 \pm 14$ & $51 \pm 9$ & $29 \pm 6$ & $7 \pm 4$ & 0 \\
\hline 4 & $1586 \pm 98$ & $1002 \pm 8$ & $13 \pm 6$ & $4 \pm 3$ & 0 & 0 \\
\hline 5 & $1003 \pm 16$ & $989 \pm 9$ & $38 \pm 5$ & $28 \pm 13$ & $12 \pm 3$ & 0 \\
\hline 6 & $1490 \pm 42$ & $987 \pm 9$ & $8 \pm 3$ & $3 \pm 1$ & 0 & 0 \\
\hline Hydroxyurea & $808 \pm 36$ & $560 \pm 13$ & $12 \pm 3$ & $7 \pm 1$ & $6 \pm 4$ & 0 \\
\hline Sulfadiazine & $489 \pm 23$ & $548 \pm 9$ & $389 \pm 34$ & $28 \pm 3$ & $12 \pm 3$ & 0 \\
\hline
\end{tabular}

Data are reported as means \pm SD for three independent experiments. Each compound was incubated, in an independent experiment, in the cultures previously infected for $24 \mathrm{~h}$, at concentrations of $0.1,1,4,8$, and $20 \mathrm{mM}$. 
was more effective against intracellular parasites than the host cells. Similar values were obtained with HU. However, sulfadiazine caused a decrease in intracellular tachyzoites (LD50 at $8 \mathrm{mM}$ ) with values near to those observed for toxicity in the host cells (LD50 at $4 \mathrm{mM}$ ). In contrast to data in Tables 1 and 2, a 1-mM concentration of the drugs was used in all experiments described below.

Table 2. LD50 (4 mM) of compounds 1-6, hydroxyurea and sulfadiazine on host cells and intracellular Toxoplasma gondii tachyzoites determined by non-linear regression.

\begin{tabular}{lcc}
\hline Compound & Host cells & Intracellular tachyzoites \\
\hline 1 & $10 \pm 0.06$ & $0.5 \pm 0.001$ \\
2 & $>20$ & $0.5 \pm 0.002$ \\
3 & $>20$ & $0.6 \pm 0.003$ \\
4 & $10 \pm 0.06$ & $0.2 \pm 0.001$ \\
5 & $>20$ & $0.5 \pm 0.003$ \\
6 & $>20$ & $0.7 \pm 0.005$ \\
Hydroxyurea & $>20$ & $0.05 \pm 0.005$ \\
Sulfadiazine & $8 \pm 0.5$ & $2 \pm 1$ \\
\hline
\end{tabular}

Data are reported as means \pm SD .
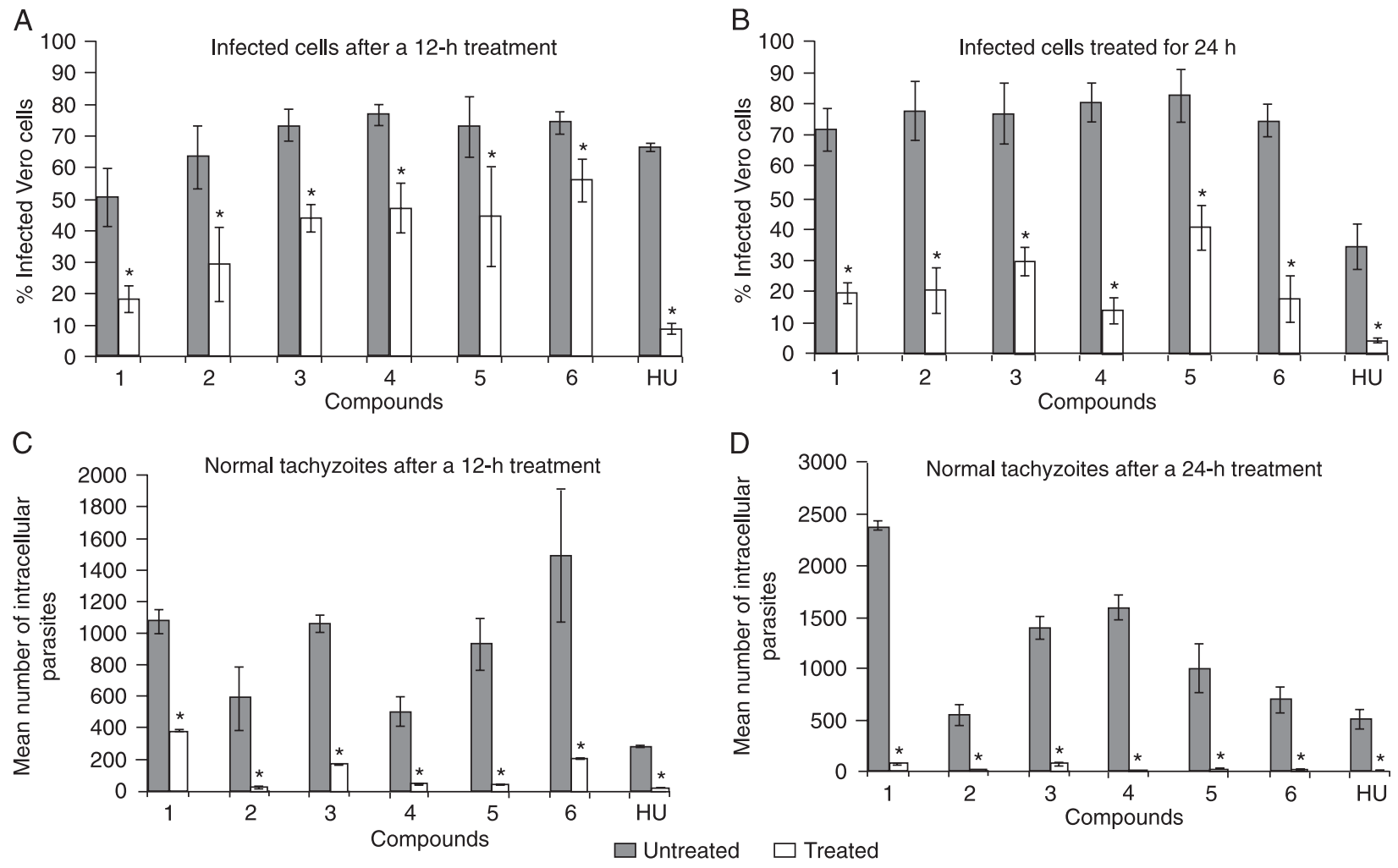

Figure 2. Survival of infected cells treated with $1 \mathrm{mM}$ compounds 1-6 and $4 \mathrm{mM}$ hydroxyurea (HU) for $12 \mathrm{~h}(\mathrm{~A})$ or $24 \mathrm{~h}(\mathrm{~B})$. Mean number of normal intracellular tachyzoites after treatment for $12 \mathrm{~h}(\mathrm{C})$ or $24 \mathrm{~h}(\mathrm{D})$. Data are reported as means \pm SD for three independent experiments. $\mathrm{P}<005$ compared to untreated culture (two-way ANOVA).

After treatment with the TSC and 4-TZD derivatives, microscopy revealed that intravacuolar parasites became altered during treatment.

In the untreated cultures, infected cells contained parasites with normal morphology in a crescent shape, multiplying inside the PV. The percentage of infected cells and the mean number of parasites are shown in Figure 2. In the presence of TSC (compounds 1-3) and 4-TZD (compounds 4-6; Figure 1), the percentage of infected cells containing normal parasites decreased after treatment for $12 \mathrm{~h}$ (Figure 2A) and even more so after $24 \mathrm{~h}$ (Figure 2B). Infection was also reduced by incubation with $\mathrm{HU}$ to a level similar to that observed for the new compounds (Figure 2). HU used for comparison $(11,12)$ was $4 \mathrm{mM}$ and other compounds were $1 \mathrm{mM}$. The reduction in infection was closely associated with the disorganization and elimination of normal parasites after $12 \mathrm{~h}$ of treatment, at which point morphologically normal tachyzoites were greatly decreased in number (Figure 2C). After $24 \mathrm{~h}$ they were completely absent in the presence of some of the compounds (Figure 2D).

Tachyzoites in the untreated culture formed a rosette arrangement and had a crescent shape (Figure 3A). During treatment with all compounds, these intravacuolar parasites showed changes such as a rounded shape and

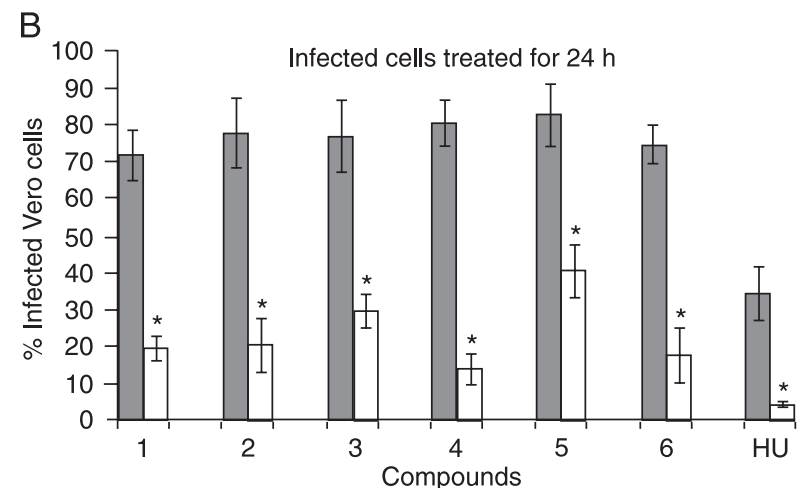




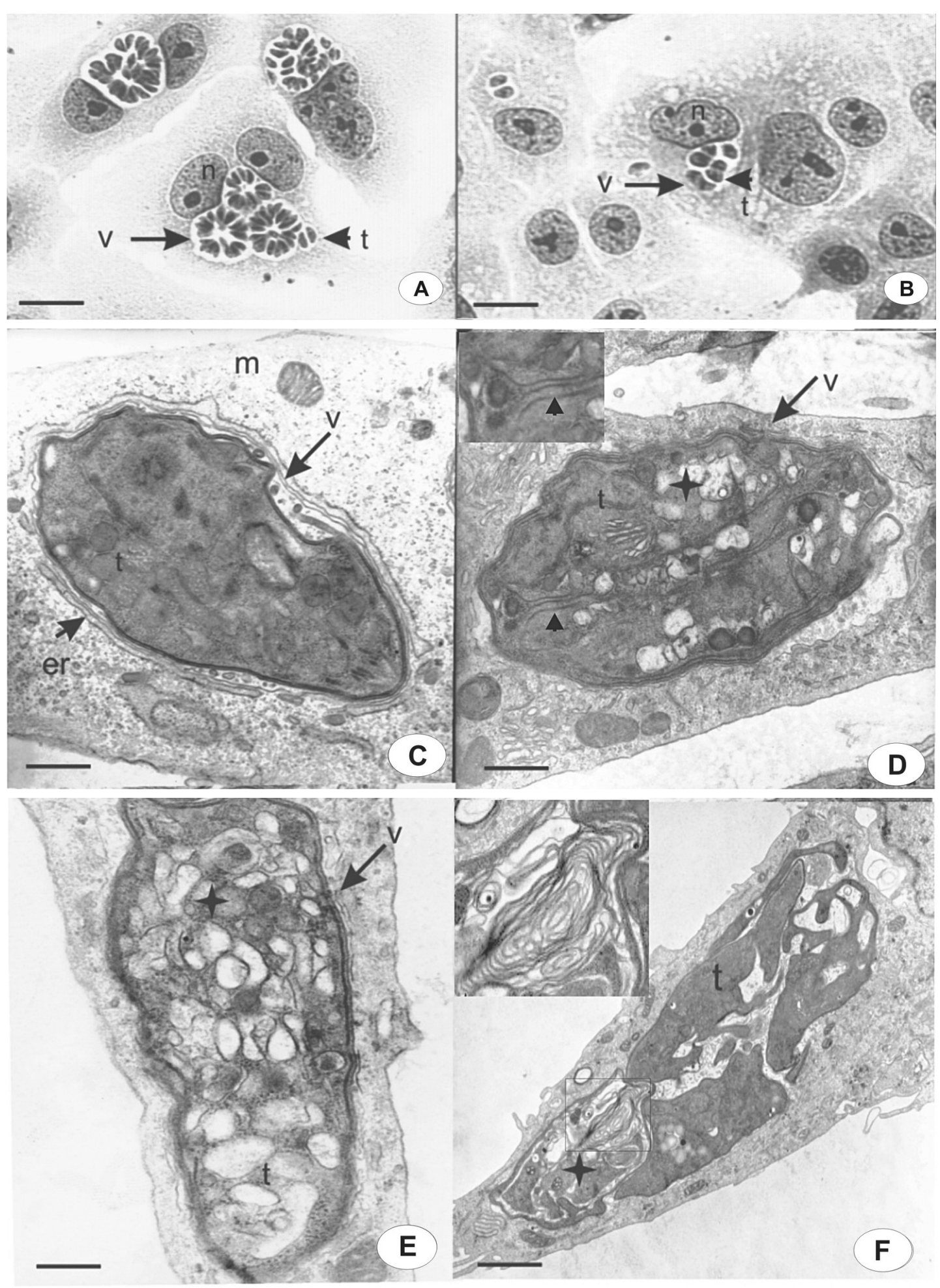

Figure 3. Morphological aspects of intravacuolar parasites observed by light microscopy $(A, B)$ or transmission electron microscopy $(\mathrm{C}-\mathrm{F})$. Untreated cells $(\mathrm{A}, \mathrm{C})$; cells treated for $12 \mathrm{~h}$ with compound $1(\mathrm{D})$; cells treated for $24 \mathrm{~h}$ with compound 2 (B,F), and $24 \mathrm{~h}$ with compound $3(E)$. Parasitophorous vacuole $(v)$; tachyzoites $(t)$; host cell nucleus $(n)$; host cell mitochondria $(m)$; endoplasmic reticulum (er); vesiculation (D, E - star); daughter parasites inner membrane complex (D - arrowhead inset) and myelin ( $F$ - star and inset). Bars: Figure A,B: $20 \mu \mathrm{m}$; Figure C-F: $1 \mu \mathrm{m}$. 
the disruption of the rosette arrangement (Figure 3B). PVs containing altered or disorganized parasites were quantified in order to demonstrate the destruction of tachyzoites (Figure 4). Parasites were unchanged in untreated cultures. Thus, the number of vacuoles containing altered parasites was determined only in the treated cultures. The number of infected cells (Figure 2A,B) with vacuoles containing disorganized and/or disrupted parasites progressively decreased between 12 and $24 \mathrm{~h}$, leading to the elimination of tachyzoites from the host cell. Differences observed among the compounds in terms of the decrease in vacuoles containing altered parasites (Figure 4) suggest that elimination of these vacuoles is closely associated with the efficiency of the anti-parasite compounds. Overall, all compounds decreased the infection in a similar way, except compounds 3 and 4, which induced a significant decrease of the number of these PVs, suggesting that these disrupted parasites had been eliminated from the host cell (Figure 4). In addition, none of the compounds used here caused drastic cytotoxic effects to the host cell, as demonstrated by the Trypan blue test (data not shown).

T. gondii has specialized organelles such as micronemes, rhoptries and dense granules that are distributed in the apical region, and an inner membrane complex and the outer plasma membrane (Figure 3C). These features changed in the presence of TSC and TZD, as demonstrated in Figure 3D-F, being similar for all compounds. The main alteration was a progressive vesiculation in the cytoplasm of the parasite (Figure 3D,E) after $12 \mathrm{~h}$ of treatment. This figure shows daughter tachyzoites that did not complete multiplication (Figure 3D, arrowhead in inset). However, secretory organelles, such as dense granules and rhoptries, were present. After incubation for $24 \mathrm{~h}$, increasing vesiculation was observed inside the tachyzoites (Figure 3E). Some vacuoles within the parasite's cytoplasm had dense and amorphous materials, and secretory organelles were not visible (Figure 3E). The vesiculation process was a consequence of the arrest of the cell cycle in the parasites and could involve secretory vesicle disorganization, features that are vital for the survival of intracellular parasites. However, different degrees of ultrastructural disorganization were seen inside the same $P V$, suggesting an asynchronous effect on tachyzoite development (Figure 3F). In this micrograph, we also observed parasite multilamellar structures inside their cytoplasm, such as the myelin-like figure (Figure 3F, inset). This same parasite had mitochondria, but secretory organelles were not observed. The vesiculation observed in the parasites was not seen in the host cells (Figure $3 \mathrm{C}-\mathrm{F}$ ), in which mitochondria and endoplasmic reticulum elements were seen around the PV but were not more highly associated with the PV membrane (Figure 3D-F), suggesting interruption of the close association between PV-containing parasites and host cell organelles.

To determine whether incubation with the compounds had a reversible anti-Toxoplasma effect, a reversibility assay was performed. Infected monolayers were incubated with compounds 1 and 4 for 12 and $24 \mathrm{~h}$, the medium was then removed and the cultures were incubated for another $24 \mathrm{~h}$ with fresh medium. After $12 \mathrm{~h}$ of treatment parasites that had a normal morphology resumed their multiplication once the drugs were removed (Figure 5A,B). The number of parasites increased significantly after the reversible assay with cultures treated for $12 \mathrm{~h}$ (Figure 5B). After $24 \mathrm{~h}$ of treatment, an increase in intracellular parasites occurred with compound 1 but infection did not increase (Figure 5B). The number of PVs containing altered parasites was similar in all reversibility assays, except for compound 1 at $24 \mathrm{~h}$, which showed a decrease related to time of culture treatment (Figure 5C). These observations could be the result of asynchronous infection, in which parasites are present at

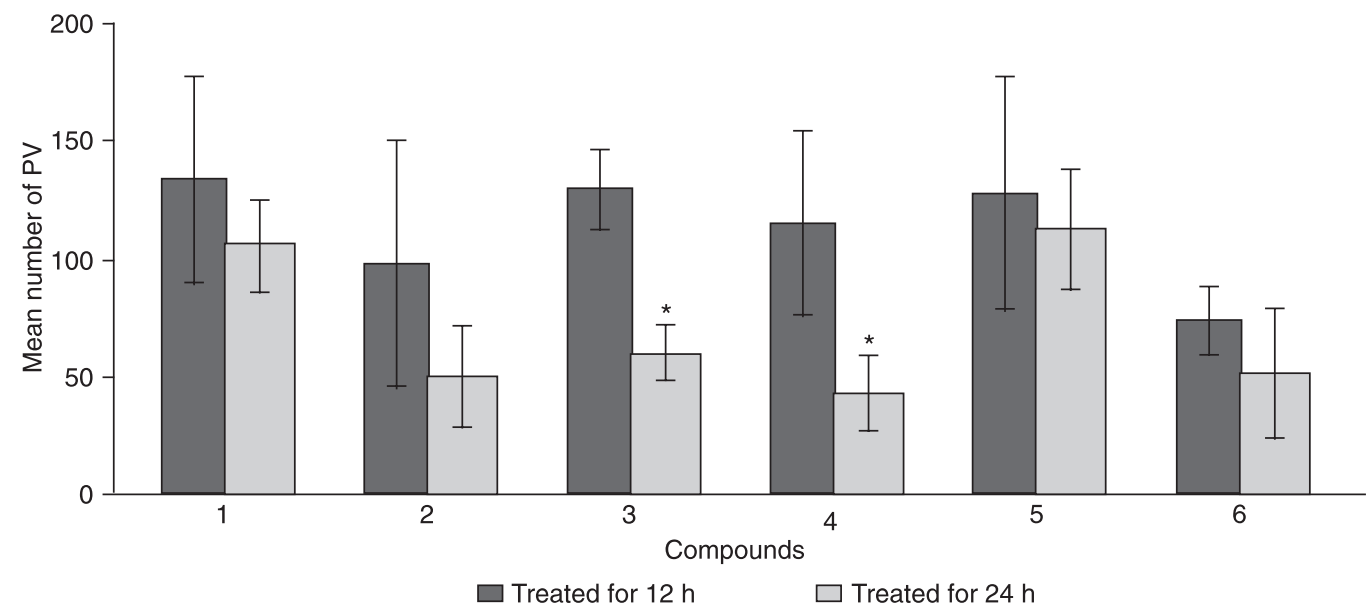

Figure 4. Mean number of parasitophorous vacuoles (PV) in infected treated culture for $12 \mathrm{~h}$ (dark gray columns) and $24 \mathrm{~h}$ compounds 1-6 (light gray columns). Data are reported as means \pm SD. ${ }^{*} \mathrm{P}<0.01$ compared to $12 \mathrm{~h}$ of treatment (two-way ANOVA). 
several steps of the cell cycle. However, since the parasites were altered during treatment, this effect was not reversible and the parasites were unable to multiply.

Although the normal parasites resumed their cell cycle, the rosette structure was rarely observed (Figure 6A,B). Abnormal intravacuolar tachyzoites remained in the culture during treatment for $12 \mathrm{~h}$ and showed disorganized morphological aspects, as illustrated in Figure 6A. However, PV-containing parasites were eliminated, or only a few remained in the host cell cytoplasm (Figure 6B). The different stages of the destruction process were evident in a single vacuole, as shown in Figure 6. Tachyzoites showed progressive ultrastructural disorganization after $12 \mathrm{~h}$ of treatment. These data support the view that compounds of this class arrest the parasite's cycle and have their antiparasite effects only in determinate phases of parasite division and development.

We showed the possible processes responsible for intravacuolar elimination of parasites in the presence of the compounds. In untreated cultures, the PV did not stain with $\mathrm{AO}$, confirming the non-acidic nature of this compartment (Figure 7A,B). By using $A O$ in cultures treated with compound 2, we observed positive vesicles in the host cells and in the PV, an indication that acidification had occurred in this environment (Figure 7C,D). In addition, the vesicles in the host cell cytoplasm were stained more strongly than the PV. The AO-positive vacuoles contained disorganized parasites (Figure 7D), whereas the untreated culture con-
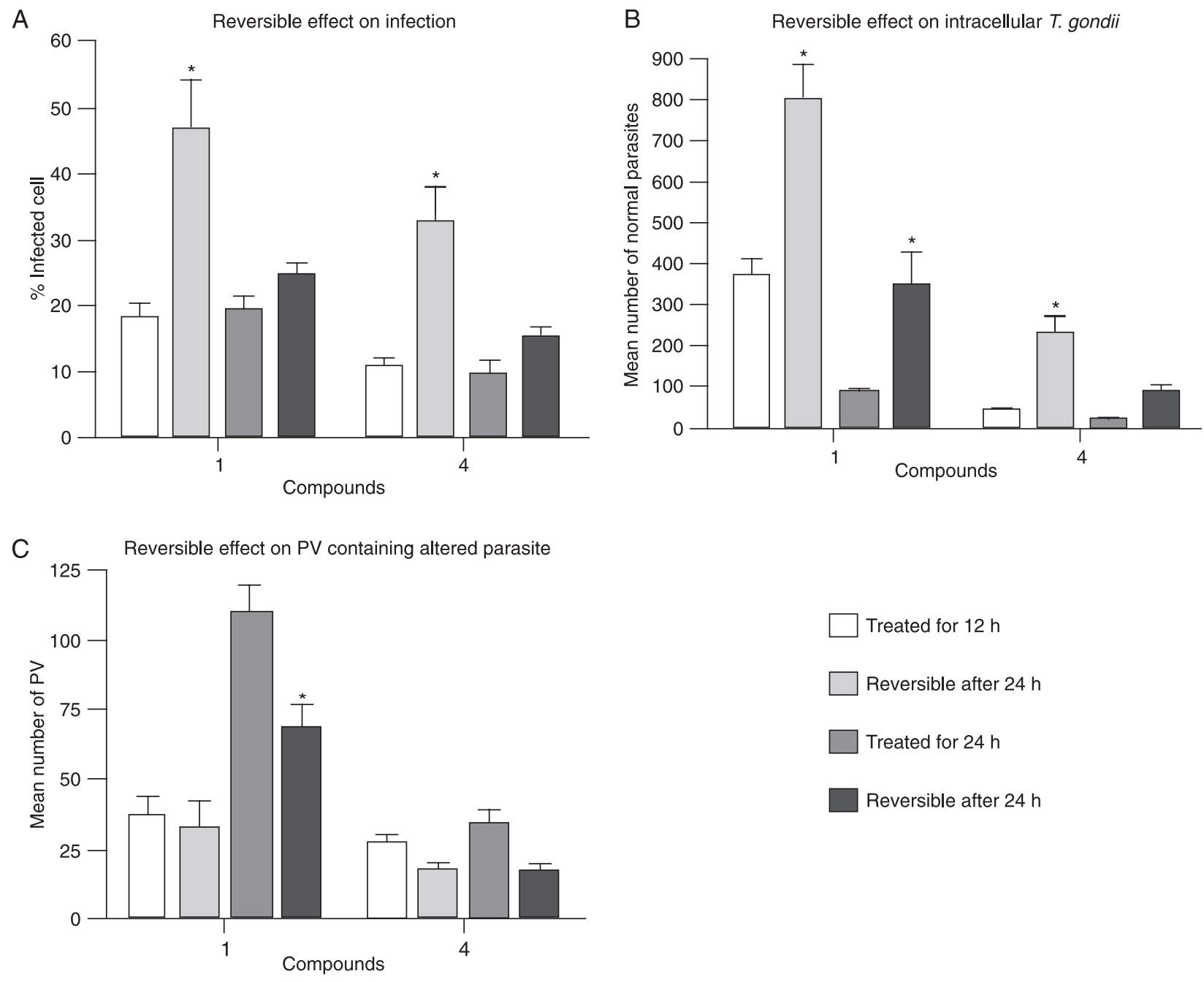

Figure 5. The reversible effects of compounds 1 and 4 were quantified after 12 or $24 \mathrm{~h}$ of treatment. Infected cultures were treated for 12 or $24 \mathrm{~h}$ and then incubated for an additional $24 \mathrm{~h}$ without drugs. The reversible effect was examined in the infected cells (A); normal intracellular parasites (B), and parasitophorous vacuoles (PV) containing altered parasites (C). Data are reported as means \pm SD of two independent experiments. ${ }^{*} \mathrm{P}<0.005$ compared to infected and treated culture (two-way ANOVA). 
tained parasites forming rosette structures. The acidification is probably caused by lysosome fusion, as demonstrated after HU treatment (25).

\section{Discussion}

Standard treatment of toxoplasmosis is accompanied by severe side effects; thus, alternative therapeutic compounds are needed. The present study was performed to evaluate the efficacy of new thiosemicarbazone against $T$. gondii. The TSC scaffold has advantages in the synthesis of new compounds, such as low molecular weight, good hydrogen bond donating and accepting capabilities, and easy, economical synthesis routes.
Recent research has demonstrated the efficacy of TSC compounds associated with metal, such as platinum, against Trypanosoma cruz, with potent activity against cruzain (26). Others showed that metronidazole-thiosemicarbazone compounds were highly effective for Entamoeba histolytica (27) and Plasmodium spp (28). Despite the wide pharmacological range of this class of compounds, drugs used here preferentially affected intravacuolar tachyzoites of $T$. gondii.

The compounds used here have $\mathrm{H}, \mathrm{Cl}$ or $\mathrm{NO}_{2}$ groups on the arylhydrazone moiety. This chemical structure showed anti-parasitic effects similar to those described by Tenório et al. (14). The best compound was acid [2-(benzylidenehydrazone)-4-oxo-1,3-thiazolidin-5-yl]acetic, that had an

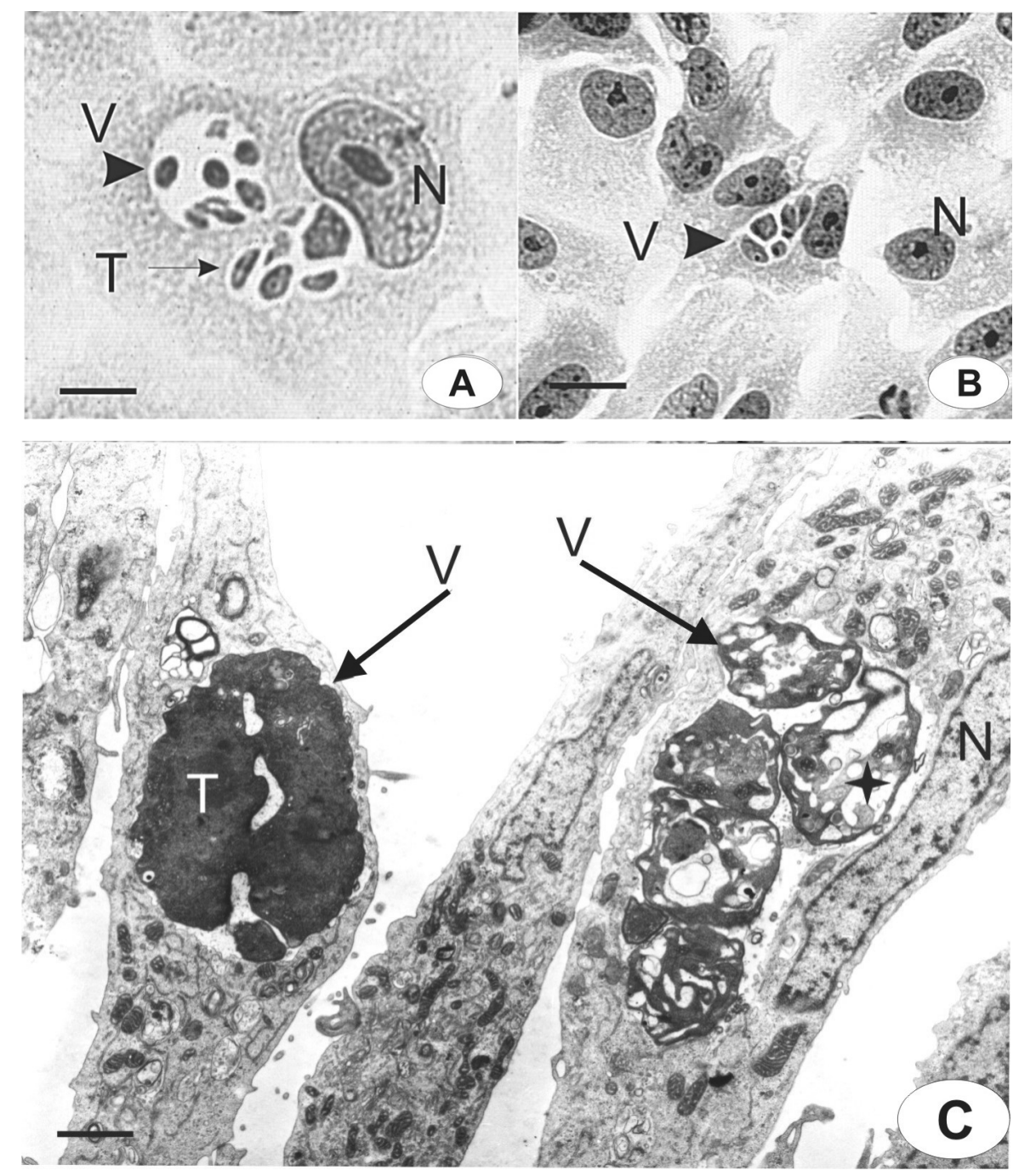

Figure 6. The reversible effects of compounds 1 and 4 were analyzed by light (A,B) or transmission electron microscopy (C). After 24 h of treatment with compound $1(A)$ and $4(B, C)$ the cultures were incubated without drugs for a further 24 h. Normal tachyzoites $(T)$ and vacuoles containing altered parasites $(V)$ were observed in the absence of the drug $(A, C)$. $N=$ host cell nucleus; star = parasite with high vacuolization. Bars: Figure A,B: $20 \mu \mathrm{m}$; Figure C: $2 \mu \mathrm{m}$. 
LD50 of $0.2 \mathrm{mM}$, after $24 \mathrm{~h}$ of treatment, without toxic effects on the host cell. This should be a good candidate for in vivo tests.

The mechanism of action of TSC and TZD compounds is mainly associated with ribonucleotide reductase inhibition, as is that of HU (29), but it may also involve inhibition of other metallic proteins involved in metabolic processes.
The ability of TSC to arrest the cell cycle has been used in a variety of situations, such as antitumoral, antiviral, and anti-protozoa treatments $(29,30)$. Another TSC, Triapine ${ }^{\circledR}$, has also shown significant activity on tumor cells (31) and was tested in a phase I clinical trial. Melo and Beiral (12) demonstrated that $\mathrm{HU}$ was efficient in arresting the multiplication of Leishmania amazonensis and Trypanosoma cruzi.
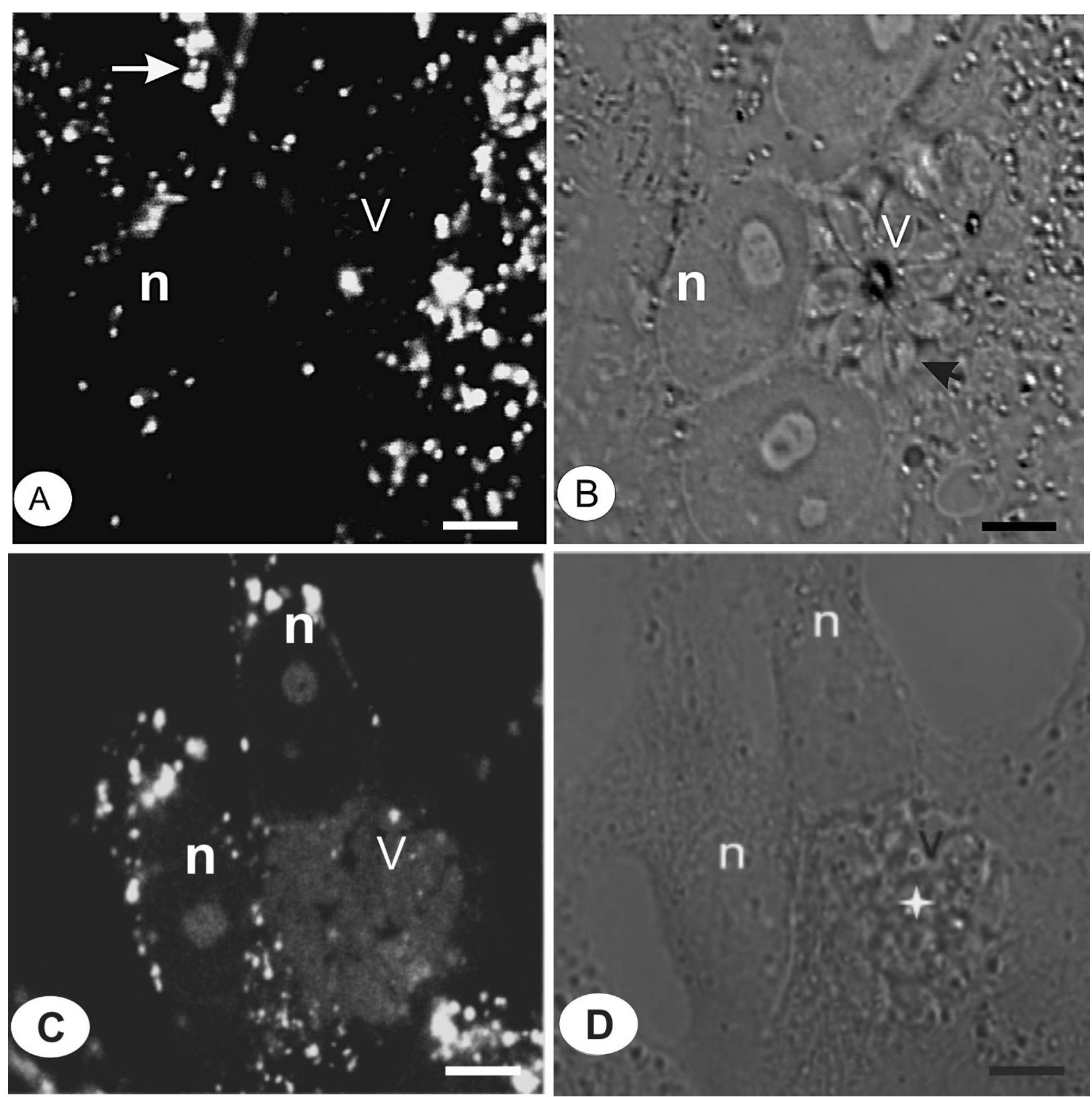

Figure 7. Vacuolar acidification (A, arrow) was observed by acridine orange staining in untreated $(A, B)$ or treated cells (C,D). A parasitophorous vacuole $(\mathrm{V})$ containing tachyzoites $(B$, arrowhead) in the control culture was not positive for this dye. After treatment with compound 2 for $24 \mathrm{~h}$ the vacuoles were acidic $(C)$ and parasites were disorganized (D, star). $\mathrm{n}=$ host cell nucleus. Bar: $10 \mu \mathrm{m}$. 
Due to their intrinsic metal (e.g., iron) chelating properties, the mechanism of action of TSC is also believed to result from the generation of reactive oxygen radical (28). In relation to $T$. gondii destruction, the present study described for the first time the main morphological features associated with TSC action.

During incubation with these drugs progressive tachyzoite disorganization and destruction were observed. This was demonstrated by the number of PV containing progressively disorganized parasites before the elimination process, and normal parasite recovery was observed in the absence of the drug, strongly suggesting that these compounds must be acting on a specific stage of the parasite's cell cycle, as also observed for HU. We also observed intense vesiculation in the parasites during incubation with the drugs and the development of myelin figures, which are associated with cell death in the parasite. Myelin figures are polar lipid-rich structures containing cholesterol, phosphatidylcholine, and sphingomyelin (32), which are capable of activating numerous metabolic pathways, especially those involved in apoptosis. In addition, the entire vesiculation process occurred in the secretory system of the parasite, which has a vital biological activity for tachyzoite survival and development.

Some studies have already explored the morphological features associated with Toxoplasma death. Ling et al. (33) described a series of intracellular mechanisms used by primed macrophage to destroy $T$. gondii. They showed that this mechanism occurs frequently at early stages of infection, and is characterized by changes of the parasitophorous vacuole membrane and parasite disorganization, which lead to autophagic processes and lysosomal degradation. A recent study by our group (25) also showed that the disorganization and destruction of intravacuolar Toxoplasma in the presence of $\mathrm{HU}$, using non-macrophage cells, involved fusion between the lysosomes of host cells and the vacuole containing the parasites. These cellular

\section{References}

1. de Melo EJT, de Carvalho TU, de Souza W. Penetration of Toxoplasma gondii into host cells induces changes in the distribution of the mitochondria and the endoplasmic reticulum. Cell Struct Funct 1992; 17: 311-317.

2. Sinai AP, Webster $P$, Joiner KA. Association of host cell endoplasmic reticulum and mitochondria with the Toxoplasma gondii parasitophorous vacuole membrane: a high affinity interaction. J Cell Sci 1997; 110 (Part 17): 2117-2128.

3. Joiner KA, Fuhrman SA, Miettinen HM, Kasper LH, Mellman I. Toxoplasma gondii: fusion competence of parasitophorous vacuoles in Fc receptor-transfected fibroblasts. Science 1990; 249: 641-646.

4. Mordue DG, Hakansson S, Niesman I, Sibley LD. Toxoplasma gondii resides in a vacuole that avoids fusion with host cell endocytic and exocytic vesicular trafficking pathways. Exp Parasitol 1999; 92: 87-99.

5. Gavin MA, Wanko T, Jacobs L. Electron microscope stud- aspects demonstrate that the PV becomes permissive to lysosome fusion when the parasite is dead after the PV has formed and developed. On the other hand, death due to pyrimethamine does not lead to PV fusion with lysosomes (3). Similar to $\mathrm{HU}$, the parasite death resulting from incubation with TSC and TZD led to vacuolar acidification, preventing tachyzoites from developing in the PV. This event is associated with elimination of the parasite by the digestive pathway of the host cell - a major mechanism of cell defense against microorganism invasion.

During treatment, the host cell organelles associated with PV-containing tachyzoites, mainly mitochondria and endoplasmic reticulum, were abolished. These results conflict with those obtained by Sinai and Joiner (34), which demonstrated that the mitochondria remain joined to the $P V$ after treatment of intracellular T. gondii with pyrimethamine, an anti-metabolic drug. The mitochondrial association with $\mathrm{PV}$ was demonstrated to involve the rhoptry protein ROP2, which exposes its amino-terminus containing an amphipathic helix reminiscent of mitochondrial import sequences (35). It would be interesting to investigate whether this molecular association is lost during treatment with antimetabolic or antiproliferative drugs.

Overall, we consider the results obtained so far to be promising since they provide new and relevant data about thiosemicarbazones and their specific action on the Apicomplexa parasite. The efficiency of TSC compounds in interrupting cell multiplication supports the potential extensive application of TSC and TZN to antiparasite therapy.

\section{Acknowledgments}

Research supported by FAPERJ. C.S. Carvalho was the recipient of a research fellowship from Universidade Estadual do Norte Fluminense (UENF). ies of reproducing and interkinetic Toxoplasma. J Protozool 1962; 9: 222-234.

6. Hu K, Roos DS, Murray JM. A novel polymer of tubulin forms the conoid of Toxoplasma gondii. J Cell Biol 2002; 156: 1039-1050.

7. de Melo EJT, de Souza W. Pathway of C6-NBD-Ceramide on the host cell infected with Toxoplasma gondii. Cell Struct Funct 1996; 21: 47-52.

8. Charron AJ, Sibley LD. Host cells: mobilizable lipid resources for the intracellular parasite Toxoplasma gondii. J Cell Sci 2002; 115: 3049-3059.

9. Sehgal A, Bettiol S, Pypaert M, Wenk MR, Kaasch A, Blader $I J$, et al. Peculiarities of host cholesterol transport to the unique intracellular vacuole containing Toxoplasma. Traffic 2005; 6: 1125-1141.

10. Hoagland C. Hematologic complications of cancer chemotherapy. In: Perry MC (Editor), Chemotherapy source book. 
Baltimore: Williams \& Wilkins, 1992. p 498-507.

11. de Melo EJT, Mayerhoffer RO, de Souza W. Hydroxyurea inhibits intracellular Toxoplasma gondii multiplication. FEMS Microbiol Lett 2000; 185: 79-82.

12. Melo EJT, Beiral HJ. Effect of hydroxyurea on the intracellular multiplication of Toxoplasma gondii, Leishmania amazonensis and Trypanosoma cruzi. Braz J Med Biol Res 2003; 36: 65-69.

13. Gwilt PR, Tracewell WG. Pharmacokinetics and pharmacodynamics of hydroxyurea. Clin Pharmacokinet 1998; 34: 347-358.

14. Tenorio RP, Carvalho CS, Pessanha CS, de Lima JG, de Faria AR, Alves AJ, et al. Synthesis of thiosemicarbazone and 4-thiazolidinone derivatives and their in vitro antiToxoplasma gondii activity. Bioorg Med Chem Lett 2005; 15: 2575-2578.

15. Bonde CG, Gaikwad NJ. Synthesis and preliminary evaluation of some pyrazine containing thiazolines and thiazolidinones as antimicrobial agents. Bioorg Med Chem 2004; 12 : 2151-2161.

16. Rao A, Balzarini J, Carbone A, Chimirri A, De Clercq E, Monforte AM, et al. Synthesis of new 2,3-diaryl-1,3-thiazolidin-4ones as anti-HIV agents. Farmaco 2004; 59: 33-39.

17. Vigorita MG, Ottana R, Monforte F, Maccari R, Monforte MT, Trovato A, et al. Chiral 3,3'-(1,2-ethanediyl)-bis[2-(3,4dimethoxyphenyl)-4-thiazolidinones] with anti-inflammatory activity. Part 11: evaluation of COX-2 selectivity and modelling. Bioorg Med Chem 2003; 11: 999-1006.

18. Ergenc N, Capan G, Gunay NS, Ozkirimli S, Gungor M, Ozbey S, et al. Synthesis and hypnotic activity of new 4-thiazolidinone and 2-thioxo-4,5-imidazolidinedione derivatives. Arch Pharm 1999; 332: 343-347.

19. Alves AJ, Leite AC, De Santana DP, Beltrao TM, Coelho MR, Gayral P. Synthesis of some 4-oxo-delta 2-thiazolin2-ylhydrazones as potential antiprotozoal agents. Farmaco 1993; 48: 1167-1171.

20. Seebacher W, Brun R, Weis R. New 4-aminobicyclo[2.2.2] octane derivatives and their activities against Plasmodium falciparum and Trypanosoma b. rhodesiense. Eur J Pharm Sci 2004; 21: 225-233.

21. Djurkovic-Djakovic O, Nikolic T, Robert-Gangneux F, Bobic B, Nikolic A. Synergistic effect of clindamycin and atovaquone in acute murine toxoplasmosis. Antimicrob Agents Chemother 1999; 43: 2240-2244.

22. Chirgwin K, Hafner R, Leport C, Remington J, Andersen J, Bosler EM, et al. Randomized phase II trial of atovaquone with pyrimethamine or sulfadiazine for treatment of toxoplasmic encephalitis in patients with acquired immunodeficiency syndrome: ACTG 237/ANRS 039 Study. AIDS Clinical Trials Group 237/Agence Nationale de Recherche sur le SIDA, Essai 039. Clin Infect Dis 2002; 34: 1243-1250.
23. Montoya JG, Liesenfeld O. Toxoplasmosis. Lancet 2004; 363: 1965-1976.

24. Kielian MC, Cohn ZA. Phagosome-lysosome fusion. Characterization of intracellular membrane fusion in mouse macrophages. J Cell Biol 1980; 85: 754-765.

25. Carvalho CS, Melo EJT. Acidification of the parasitophorous vacuole containing Toxoplasma gondii in the presence of hydroxyurea. An Acad Bras Cienc 2006; 78: 475-484.

26. Vieites M, Otero L, Santos D, Toloza J, Figueroa R, Norambuena E, et al. Platinum(II) metal complexes as potential anti-Trypanosoma cruzi agents. J Inorg Biochem 2008; 102 : 1033-1043.

27. Abid M, Agarwal SM, Azam A. Synthesis and antiamoebic activity of metronidazole thiosemicarbazone analogues. Eur J Med Chem 2008; 43: 2035-2039.

28. Biot C, Pradines B, Sergeant MH, Gut J, Rosenthal PJ, Chibale K. Design, synthesis, and antimalarial activity of structural chimeras of thiosemicarbazone and ferroquine analogues. Bioorg Med Chem Lett 2007; 17: 6434-6438.

29. Casas JS, Garcia-Tasende MS, Sordo J. Main group metal complexes of semicarbazones and thiosemicarbazones. A structural review. Coord Chem Rev 2000; 209: 197-261.

30. Bharti N, Husain K, Gonzalez Garza MT, Cruz-Vega DE, Castro-Garza J, Mata-Cardenas BD, et al. Synthesis and in vitro antiprotozoal activity of 5-nitrothiophene-2-carboxaldehyde thiosemicarbazone derivatives. Bioorg Med Chem Lett 2002; 12: 3475-3478.

31. Finch RA, Liu MC, Cory AH, Cory JG, Sartorelli AC. Triapine(3-aminopyridine-2-carboxaldehydethiosemicarbazone; 3-AP): an inhibitor of ribonucleotide reductase with antineoplastic activity. Adv Enzyme Regul 1999; 39: 3-12.

32. Vejux A, Kahn E, Dumas D, Bessede G, Menetrier F, Athias $A$, et al. 7-Ketocholesterol favors lipid accumulation and colocalizes with Nile Red positive cytoplasmic structures formed during 7-ketocholesterol-induced apoptosis: analysis by flow cytometry, FRET biphoton spectral imaging microscopy, and subcellular fractionation. Cytometry A 2005; 64: 87-100.

33. Ling YM, Shaw MH, Ayala C, Coppens I, Taylor GA, Ferguson DJ, et al. Vacuolar and plasma membrane stripping and autophagic elimination of Toxoplasma gondii in primed effector macrophages. J Exp Med 2006; 203: 2063-2071.

34. Sinai AP, Joiner KA. The Toxoplasma gondii protein ROP2 mediates host organelle association with the parasitophorous vacuole membrane. J Cell Biol 2001; 154: 95-108.

35. Beckers CJ, Dubremetz JF, Mercereau-Puijalon O, Joiner KA. The Toxoplasma gondii rhoptry protein ROP 2 is inserted into the parasitophorous vacuole membrane, surrounding the intracellular parasite, and is exposed to the host cell cytoplasm. J Cell Biol 1994; 127: 947-961. 